\title{
Neutrinos as a signature of ultrahigh energy photons at high red shift
}

\author{
Marieke Postma \\ Department of Physics and Astronomy, UCLA, Los Angeles, CA 90095-1547
}

(October 25, 2018)

\begin{abstract}
Sources of ultrahigh energy photons operating at high red shift produce a diffuse background of neutrinos. At high red shift, when the cosmic microwave background radiation has a higher temperature, an electromagnetic cascade originated by an energetic photon can generate neutrinos via muon and pion production and decay. We have calculated numerically the neutrino spectrum produced by various photon sources. A distinctive feature of the produced flux is a "bump" in the spectrum at neutrino energies $E \sim 10^{17} \mathrm{eV}$. The produced flux is largest for $m=3$ sources (e.g necklaces), with $E^{2} J(E) \sim 10 \mathrm{eVcm}^{-2} \mathrm{~s}^{-1} \mathrm{sr}^{-1}$ at these energies. Detection of such neutrinos can help understand the origin of ultrahigh energy cosmic rays.
\end{abstract}

PACS numbers: 98.70.Sa, 95.85.Ry, 98.70.Vc $\quad$ UCLA/01/TEP/2

\section{INTRODUCTION}

In the near future several experiments will become sensitive to small fluxes of very high energy neutrinos. One may hope to observe neutrinos from active galactic nuclei (AGN), gamma ray bursts, other astrophysical sources, and, possibly, from yet undiscovered objects that have emerged from particle theory, e.g., topological defects (TD). To learn new physics from the future observations, it is important to identify signatures of certain sources that may contribute to diffuse neutrino flux. In this paper we detail the description of one such signature [1], namely the imprint of ultrahigh energy photons from high red shift on the neutrino spectrum. At high red shift, when the cosmic microwave background radiation (CMBR) has a higher temperature, an electromagnetic cascade originated by an ultrahigh energy photon can generate neutrinos via muon and pion production and decay. The present-day energies of such neutrinos are about $10^{17} \mathrm{eV}$. Detection of a "bump" in the neutrino spectrum at $10^{17} \mathrm{eV}$ can indicate the presence of ultrahigh energy photon sources at high red shift.

The question of ultrahigh energy photon sources was brought in sharp focus by recent observations of cosmic rays [2] with energies beyond the Greisen-ZatsepinKuzmin (GZK) cutoff [3]. The origin of such cosmic rays remains an outstanding puzzle 断. Most of the proposed explanations can be categorized in two main classes. In the bottom-up/acceleration scenarios, charged particles are accelerated to ultrahigh energies in giant astrophysical "accelerators", such as active galactic nuclei and radio galaxies [5].6]. In the top-down/decay scenario on the other hand, massive objects such as topological defects [17 10] and superheavy relic particles [11 113] decay, emitting mainly ultrahigh energy photons. Topological defects and relic particles can exist at much higher red shift than the astrophysical candidate sources, which are formed only after the onset of galaxy formation.
To understand the origin of the ultrahigh energy cosmic rays (UHECR), it is crucial to distinguish between these two different scenarios. In [1] we showed that sources of ultrahigh energy photons operating at high red shift produce a diffuse background of neutrinos with energies $E_{\nu} \sim 10^{17} \mathrm{eV}$. Our rough estimates indicated that for some photon sources, e.g. necklaces, this neutrino flux is large enough to be detectable in the near future. It is therefore useful to get a better estimate of the produced neutrino flux. To this end we have done a numerical calculation. In the present paper we will present the results.

Generation of ultrahigh energy neutrinos has been studied 8 8 10,5,6, 14, 19] for various sources at small red shift, for which muon pair production can be neglected. However, a substantial flux of neutrinos could be produced at earlier times, when the propagation of photons was different from that in the present universe because the intergalactic magnetic field was weaker, the density of radio background was lower, and the cosmic microwave background density and temperature were higher. At red shift $z$ the temperature of the cosmic microwave background radiation increases by a factor $(1+z)$. Because of this, at high red shift ultrahigh energy photons and electrons can produce muons through interactions with the CMB photons. Muons decay into neutrinos. This is in sharp contrast with the $z \lesssim 1$ case, where the photons do not produce neutrinos as they lose energy mainly by scattering off the radio background and in the subsequent electromagnetic cascade 20, 4 ].

In section [1] we will describe in detail this neutrino production mechanism. We will give an estimate for the produced flux of neutrinos in section IIII; the numerical results are presented in section IV. Our discussion applies to any source of photons active at high red shift. The latter requirement excludes most astrophysical sources [5]. Topological defects and decaying relic particles on the other hand, could operate at much higher red shifts. 
These sources are expected to produce photons with energies as high as $10^{20} \mathrm{eV}$. Their presence need not be connected to the GZK puzzle. Superconducting strings and other rapidly evolving topological defects, for example, are ruled out as the origin of observed ultrahigh energy cosmic rays, because they would produce too large a flux of secondary, low-energy photons. However, despite this constraint on their present density, such sources could have existed in the early universe and could produce a detectable flux of neutrinos.

\section{NEUTRINO PRODUCTION}

The propagation of energetic photons and electrons is governed by their interactions with the cosmic background radiation. At small red shift they lose energy mainly through interactions with the radio background. The main interactions involved in the electromagnetic cascade are pair production $\gamma \gamma \rightarrow e^{+} e^{-}$and inverse Compton scattering $e \gamma \rightarrow e \gamma$. The cross section for pair production is peaked near the threshold, where $E_{\gamma} \epsilon \simeq m_{e}^{2}$. Therefore, for ultrahigh energy (UHE) photons the most effective targets are background photons with energy $\epsilon \approx m_{e}^{2} / E_{\gamma} \lesssim 10^{-6} \mathrm{eV}$, i.e., radio photons. The radio background is generated by normal and radio galaxies. Its present density 21] is higher than that of CMB photons in the same energy range. As a result, the mean free path for energetic photons and electrons is determined by the radio background, and at small red shift the main source of energy loss for UHE photons is electromagnetic cascade generated by interactions with radio photons. At red shift $z$, however, the density of CMB photons is higher by a factor $(1+z)^{3}$, while the density of radio background is either constant or, more likely, lower. Some models of cosmological evolution of radio sources 22 predict a sharp drop in the density of radio background at red shift $z \gtrsim 2$. More recent observations 23] indicate that the decrease of radio background at $z>2$ is slow. In any case, at high red shift one expects the CMB to set the mean free path and thus become the dominant source of energy loss for photons, because of the $(1+z)^{3}$ increase in the density. Let $z_{\mathrm{R}}$ be the red shift at which scattering off radio background can be neglected with respect to scattering of CMBR. Based on the analyses of Refs. [22,23], we take $z_{\mathrm{R}} \sim 5$.

Another source of energy loss is the synchrotron radiation emitted by the cascade electrons in the intergalactic magnetic field (IGMF). This is an important effect at small red shift, but it becomes less significant at earlier times when the IGMF is weak. For $z>z_{\mathrm{M}} \sim 5$ synchrotron losses are small compared to energy losses resulting from interactions with the $\mathrm{CMB}$ radiation. We will use the value $z_{\min }=\max \left(z_{\mathrm{R}}, z_{\mathrm{M}}\right) \approx 5$ in what follows. As discussed in section IV, a higher value of $z_{\min }$, even as high as 10, would not make a big difference in the flux of the signature neutrinos.

Not only the density of the CMB increases with red shift, so does its temperature: $T_{C M B}(z)=2.7(1+z) \mathrm{K}$. The center of mass energy in scatterings of UHE electrons and photons with CMB photons increases, and new, neutrino producing interactions become possible. At red shift $z>z_{\text {min }}$, where radio background and the intergalactic magnetic field can be neglected, photons and electrons scattering off the CMB can produce muons through the processes $\gamma \gamma_{C M B} \rightarrow \mu^{+} \mu^{-}$and $e \gamma_{C M B} \rightarrow$ $e \mu^{+} \mu^{-}$. Muons decay into neutrinos: $\mu \rightarrow e \nu_{e} \nu_{\mu}$. The threshold for these muon producing interactions is $\sqrt{s}>2 m_{\mu}=0.21 \mathrm{GeV}$, or

$$
E_{\gamma, e}>E_{\mathrm{th}}(z)=\frac{10^{20} \mathrm{eV}}{1+z}
$$

But will muons, and thus neutrinos, indeed be produced? To answer this question one has to look closely at the propagation of UHE photons at $z \gtrsim 5$. Photons will scatter off the CMBR; for photon energies above the threshold for muon pair production, the reactions $\gamma \gamma_{C M B} \rightarrow e^{+} e^{-}, \gamma \gamma_{C M B} \rightarrow e^{+} e^{-} e^{+} e^{-}$and $\gamma \gamma_{C M B} \rightarrow$ $\mu^{+} \mu^{-}$are possible. For $\sqrt{s}>2 m_{\pi^{ \pm}}=0.28 \mathrm{GeV}$ charged pion production may also occur. Accelerator experiments show that the pion cross section is small compared to the cross sections for muon production at the energies of interest [24]. We will, therefore, neglect it.

The cross section for electron pair production (PP) decreases with increasing photon energy

$$
\sigma_{\mathrm{PP}}=\frac{8 \pi \alpha^{2}}{s} \ln \frac{s}{m_{e}^{2}} \quad\left(s \gg m_{e}^{2}\right)
$$

while the cross section for double pair production (DPP), a higher order QED process, quickly approaches its asymptotic value [25]

$$
\sigma_{\mathrm{DPP}} \simeq \frac{172 \alpha^{4}}{36 \pi m_{e}^{2}} \simeq 6.45 \mu \mathrm{barn} \quad\left(s \gg m_{e}^{2}\right) .
$$

$\mathrm{PP}$ is the dominant reaction for photon energies $E_{\gamma} \lesssim$ $5 \times 10^{20} \mathrm{eV} /(1+z)$. Since the energies of the two interacting photons are vastly different, either the electron or the positron from PP has energy close to that of the initial photon. At higher photon energies, DPP becomes more important. One or more energetic electrons are produced in this reaction. Muon production is suppressed at all energies, its cross section being smaller than that for electron pair production by a factor 2 at threshold to a factor 10 at higher energies. Thus after an initial $\gamma \gamma_{C M B}$ reaction, there is a small chance muons are produced, but most likely one ends up with one or more UHE electrons.

These electrons continue to scatter off the CMBR. At lower energies, inverse Compton scattering, $e \gamma_{C M B} \rightarrow$ 
$e \gamma$, converts high-energy electrons into high-energy photons [4]. However, at energies above the muon threshold, higher order processes, such as triplet production (TPP) $e \gamma_{C M B} \rightarrow e e^{+} e^{-}$and electron muon-pair production (MPP) $e \gamma_{C M B} \rightarrow e \mu^{+} \mu^{-}$, dominate. For higher energies charged pion production may also occur, but we expect it to be suppressed by the same hadronic physics that suppressed pion production in the photon-photon reaction, and we will neglect it. For center of mass energies $s \gg m_{e}^{2}$, the inelasticity $\eta$ for TPP is very small [4, 26, 27]:

$$
\eta \simeq 1.768\left(s / m_{e}^{2}\right)^{-3 / 4}<10^{-3}
$$

Hence, the energy attenuation length $\lambda_{\text {eff }}$ is much larger than the TPP interaction length: $\lambda_{\mathrm{TPP}} \simeq \eta \lambda_{\mathrm{eff}}$. One of the electrons produced through TPP carries almost all $(1-\eta)$ of the incoming electron's energy. It can interact once again with the CMBR. As a result, the leading electron can scatter many times before losing a considerable amount of energy. Each time it scatters with a CMB photon there is another chance to produce a muon pair, until the electron energy decreases below threshold for muon production. To determine whether muons are produced, one must compare the energy attenuation length for triplet production with the interaction length for muon-pair production. The interaction length is given by $\lambda^{-1} \simeq\left\langle n_{C M B}\right\rangle v \sigma$, and thus the ratio is $R=\lambda_{\text {eff }} / \lambda_{\mathrm{MPP}} \simeq \sigma_{\mathrm{MPP}} /\left(\eta \sigma_{\mathrm{TPP}}\right)$. For $s \gg m_{e}^{2}$ the cross section for TPP is 26, 27.

$$
\sigma_{T P P} \simeq \frac{\alpha^{3}}{m_{e}^{2}}\left(\frac{28}{9} \ln \frac{s}{m_{e}^{2}}-\frac{218}{27}\right) .
$$

The MPP cross section in the energy range just above the threshold $5 m_{\mu}^{2}<s<20 m_{\mu}^{2}$ is of the order of $0.1-1 \mathrm{mb}$, and the ratio in this range is $R \sim 100$.

Since $\lambda_{\text {eff }} \gg \lambda_{\text {MPP }}$, in the absence of dense radio background and intergalactic magnetic fields, all electrons with $E \gtrsim E_{\text {th }}$ pair-produce muons before their energy is reduced by the cascade. For muon production close to the threshold, each muon carries on average $1 / 4$ of the incoming electron's energy [27]. Muons decay before they can interact with the photon background. Each energetic muon produces two neutrinos and an electron. The electron produced alongside the muon pair gets half or more of the incoming electron's energy; it can interact again with the CMBR to produce muons. This process can repeat until the energy of the regenerated electron decreases below the threshold for muon pair production.

\section{FLUX ESTIMATES}

Let us assume that the time dependence of the rate of photon emission can be parametrized as

$$
\dot{n}_{\gamma}\left(E_{\gamma}, t\right)=\dot{n}_{\gamma, 0}(E)\left(\frac{t}{t_{0}}\right)^{-m}
$$

The subscript 0 denotes the present-day value of the parameter. The value of $m$ depends on the source under consideration. For long lived relic particles and topological defects, the rate of photon emission is indeed (approximately) of the above form, with $m=0$ for decaying relic particles, $m=3$ for strings, monopolonium and necklaces, and $m \geq 4$ for superconducting strings. Monopolonium is a bound monopole anti-monopole pair. Necklaces are networks of strings and monopoles, with two monopoles attached to each string: the monopoles are the beads in a necklace of strings.

All photons produced at red shifts $z_{\min } \leq z \leq z_{\max }$ contribute to the present neutrino flux. Here $z_{\min } \sim 5$ is the minimum red shift for which radio background and magnetic fields are negligible, and $z_{\max }$ is the maximum red shift for which the universe is transparent to UHE neutrinos. The value of $z_{\max }$ is determined by the neutrino interactions with the relic neutrino background. The absorption red shift for neutrinos with energy $\sim 10^{17} \mathrm{eV}$ is $z_{\max } \sim 3 \times 10^{3}$ 28. The neutrino flux is:

$$
\begin{aligned}
\phi_{\nu} & =\xi \int_{z_{\min }}^{z_{\max }} \mathrm{d} t \dot{n}_{\gamma}(z)(1+z)^{-4} \\
& =\xi \frac{3}{-2 a} t_{0} \dot{n}_{\gamma, 0}\left(E>E_{\mathrm{th}}\right)\left[\left(1+z_{\min }\right)^{a}-\left(1+z_{\max }\right)^{a}\right],
\end{aligned}
$$

where $a=(3 m-11) / 2$, and $\xi$ is the number of neutrinos produced per UHE photon. An UHE photon produces an UHE electron. If energetic enough, this electron produces a muon pair, and four neutrinos are generated. However, for initial electron energies just above threshold, it may often occur that repeated TPP reactions decreases the energy below threshold before any muons are produced. Thus for photons with energies close to threshold for muon production $\xi<4$. On the other hand, for higher photon energies one can have $\xi>4$. This is because higher energetic photons are more likely to produce a double pair of electrons (DPP), i.e., produce more than one UHE electron. Furthermore, the electron produced alongside the muon pair in MPP may be energetic enough for a second round of muon pair-production. Our numerical calculation (see section [V) gives $\xi=0.6$ for $E_{\gamma}(1+z)=1 \times 10^{20} \mathrm{eV}$, $\xi=4.0$ for $E_{\gamma}(1+z)=2 \times 10^{20} \mathrm{eV}$, and $\xi=7.7$ for $E_{\gamma}(1+z)=3 \times 10^{20} \mathrm{eV}$. We will take $\xi \sim 4$ in our estimate.

Rapidly evolving sources with $m \geq 4$, such as superconducting strings, are ruled out as the origin of the observed ultrahigh energy cosmic rays. The strongest constraint on the present density of such sources comes from measurements of the diffuse $\gamma$-background below $100 \mathrm{GeV}$, 4, 9,30. UHE photons lose energy in the electromagnetic cascade involving $\mathrm{PP}$ and ICS. When the photon energy drops below PP threshold, the photon attenuation length becomes small, and for $z \lesssim 10^{3}$ the universe is transparent to these low energetic photons. 
The flux of the cascade photons in the energy range $10 \mathrm{MeV}<E<100 \mathrm{GeV}$ must be lower than the flux measured by the Energetic Gamma Ray Experiment Telescope (EGRET): $\omega_{\text {cas }} \leq 2.6 \times 10^{-6} \mathrm{eVcm}^{3}$. Although the present density of $m \geq 4$ sources is constrained, it is conceivable that in the early universe they may have existed in large enough number to produce an appreciable neutrino flux.

For $m<11 / 3, a<0$, and, according to eq. (7), the photon sources at smaller red shift, $z \sim z_{\min } \approx 5$, are the most important. But this is only true for the contribution from the most energetic photons, those with energies above the threshold at small red shift, as given by eq. (11). As $z$ increases, the threshold energy for muon production decreases, and lower energetic photons can also produce muons. Moreover, since the photon spectrum is a falling function of energy, low energy photons are more abundant; they all contribute at large red shift. Which effect wins, which red shift is favored, depends on both the evolution index $m$ and the shape of the photon spectrum. For a photon spectrum of the form $\dot{n}_{\gamma} \propto E_{\gamma}^{-\beta}$ however, the red shift dependence of the flux simplifies. Using that all photons with energy $E>E_{\text {th }}$ produce muons, the neutrino flux then becomes

$$
\phi_{\nu}=\xi \dot{n}_{0} \frac{3}{2|\beta-1|}\left(10^{20} \mathrm{eV}\right)^{1-\beta} C
$$

with

$$
C= \begin{cases}b^{-1}\left[\left(1+z_{\max }\right)^{b}-\left(1-z_{\min }\right)^{b}\right], & b \neq 0 \\ \ln \left(z_{\max } / z_{\min }\right), & b=0 .\end{cases}
$$

Here $\dot{n}_{0}$ is an overall normalization constant and $b=$ $(2 \beta+3 m-13) / 2$. For $b<0$ small red shifts are most important, whereas for $b \geq 0$ large $z$ dominates.

Nevertheless, the most energetic neutrinos are all produced at small red shift. We will estimate the flux of these high energy neutrinos, leaving the total flux for the numerical calculation. The highest energy neutrinos are produced at red shift $z \lesssim 10$. We will assume that the photon spectrum is of the form $\dot{n}_{\gamma}=\dot{n}_{0} E_{\gamma}^{-\beta}\left(t / t_{0}\right)^{m}$. If the photon sources are the origin of the UHECR today one can use the observed UHECR flux to fix the normalization constant $\dot{n}_{0}$. This gives

$$
\phi_{\nu}=\phi_{\mathrm{CR}} \xi \frac{\left(z_{\min }\right)^{b}-\left(z_{\max }\right)^{b}}{1-\left(z_{\mathrm{GZk}}, \gamma\right)^{b}}
$$

where we have used that only photons emitted at red shift $0<z<z_{G Z K, \gamma}$ contribute to the observed UHECR flux. Photons with energies $E_{\gamma} \sim 10^{20} \mathrm{eV}$ have an energy attenuation length of $\sim 10 \mathrm{Mpc}$ [4] in the present universe; this corresponds to red shift $z_{G Z_{K}, \gamma}=0.002$. The observed UHECR flux of particles with $E>E_{\mathrm{GZK}} \sim$ $5 \times 10^{19} \mathrm{eV}$ is $\phi_{\mathrm{CR}} \sim 10^{-19} \mathrm{~cm}^{-2} \mathrm{~s}^{-1} \mathrm{sr}^{-1}$. Taking $\beta=2$, in agreement with UHECR observations, and $z_{\max }=10$ we then obtain for the flux of energetic neutrinos:

$$
\phi_{\nu} \sim \begin{cases}7 \times 10^{-20} \mathrm{~cm}^{-2} \mathrm{~s}^{-1} \mathrm{sr}^{-1}, & m=0, \\ 1 \times 10^{-18} \mathrm{~cm}^{-2} \mathrm{~s}^{-1} \mathrm{sr}^{-1}, & m=1, \\ 3 \times 10^{-17} \mathrm{~cm}^{-2} \mathrm{~s}^{-1} \mathrm{sr}^{-1}, & m=2, \\ 7 \times 10^{-16} \mathrm{~cm}^{-2} \mathrm{~s}^{-1} \mathrm{sr}^{-1}, & m=3 .\end{cases}
$$

A value of $\beta=1.5$ or $\beta=2.5$ changes the produced flux by about a factor 2 . The above results are valid for photon sources that are uniformly distributed throughout the universe. Sources that cluster in galactic halos (e.g. supermassive particles) produce a lower flux, since the clustering enhances their contribution to the observed UHECR flux. To get the neutrino flux for photon sources with clustering properties, one has to divide the results in eq. (11) by the over-density of these sources in our galaxy.

The energy of the produced neutrinos at red shift $z$ is $E_{\nu}(z) \sim E_{\mu} / 3$. It is then further red shifted by a factor $(1+z)^{-1}$. Assuming a falling photon spectrum, we expect most of neutrinos to come from photons near the threshold, eq. (1]). We estimate the energy of the most energetic neutrinos after the red shift $E_{\nu} \sim 10^{17} \mathrm{eV}$.

The largest flux of neutrinos are produced by $m=3$ sources. Examples of such sources are necklaces and monopolonium. In general, topological defects emit both UHE protons and photons. When the protons, and not the photons, are responsible for the UHECR the neutrino flux can no longer be normalized as in eq. (10). Therefore, whenever we state results for necklaces or monopolonium, we will use the differential photon flux $J(E)$ calculated in [9, 17, 18 for normalization instead. For these sources, $\dot{n}_{\gamma, 0}\left(E>E_{\min }\right) \sim L^{-1} \int_{E_{\min }} \mathrm{d} E J(E)$, with $L$ the length scale from which the photons are collected. Because the photon flux is a sharply falling function of energy, $\dot{n}_{\gamma, 0}$ is dominated by photons with energies $E \sim E_{\min }$. Monopolonium clusters in galaxies and has $L \sim L_{\text {gal }} \sim 100 \mathrm{kpc}$, the size of our galaxy. Their overdensity in the galaxy is $\sim 2 \times 10^{5}[9]$. Necklaces on the other hand are distributed uniformly throughout the universe; for them $L=L_{\gamma} \sim 5 \mathrm{Mpc}$, the photon absorption length at these energies. We then obtain for the flux of neutrino produced at low red shift:

$\phi_{\nu} \sim \begin{cases}10^{-18} \mathrm{~cm}^{-2} \mathrm{~s}^{-1} \mathrm{sr}^{-1}, & \text { monopolonium }(m=3), \\ 10^{-16} \mathrm{~cm}^{-2} \mathrm{~s}^{-1} \mathrm{sr}^{-1}, & \text { necklaces }(m=3) .\end{cases}$

\section{NUMERICAL RESULTS}

We have calculated numerically the neutrino flux produced by photon sources at high red shift. According to our estimate, eq. (11), $m=3$ sources produce the largest flux of neutrinos, and we will concentrate on them. In particular, we will give a detailed discussion of the neutrino flux produced by necklaces, a candidate $m=3$ 


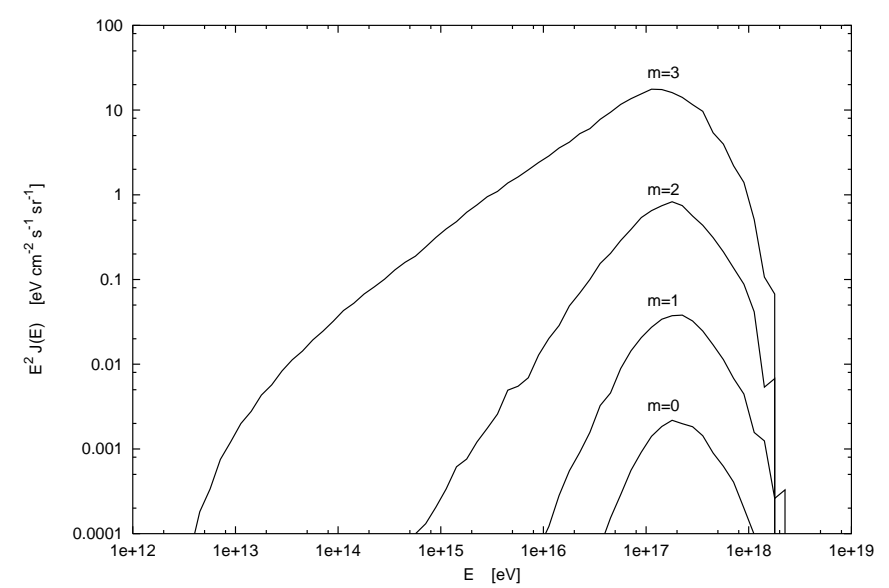

FIG. 1. Differential neutrino flux $E^{2} J(E)$ produced by sources with different evolution indices: $m=0,1,2,3$ and 4. For all plots $\beta=2, z_{\max }=10^{3}$, and $z_{\min }=5$.

source. Furthermore, we have investigated the possibility that $m=4$ sources, whose density is constrained by bounds on the low energy $\gamma$-ray flux, can nevertheless produce an observable flux of neutrinos.

We studied neutrino production using a Monte Carlo approach. In our simulation, the red shift at which the UHE photon is emitted and its energy are generated randomly with weight functions $\partial J\left(E_{\gamma}, z\right) / \partial z$ and $\partial J\left(E_{\gamma}, z\right) / \partial E_{\gamma}$ respectively. Here $J\left(E_{\gamma}, z\right)$ is the differential photon flux generated by the sources under consideration. The red shift dependence of the photon flux is due to the time evolution of the sources, given by eq. (6), and the expansion of the universe. The photon spectrum $\partial J\left(E_{\gamma}, z\right) / \partial E_{\gamma}$ is a sharply falling function of energy. It has been calculated for various topological defects and relic particles in [9, 17, 18].

Next, we let the UHE photons scatter with CMB photons, generating the scattering angle and the energy of the background photons randomly for each interaction. Radio background and intergalactic magnetic fields are insignificant for $z>z_{\min }$, and their effects are neglected. The leading particle, i.e. the particle with the highest energy produced in each interaction, is followed throughout the electromagnetic cascade until its energy becomes too low to produce muons. The probabilities for competing reactions are determined by the cross sections; which reaction actually occurs is once again determined randomly. The reactions taken into account are $\gamma \gamma_{C M B} \rightarrow e^{+} e^{-}(\mathrm{PP}), \gamma \gamma_{C M B} \rightarrow e^{+} e^{-} e^{+} e^{-}$ (DPP), $\gamma \gamma_{C M B} \rightarrow \mu^{+} \mu^{-}$(MP), $e \gamma_{C M B} \rightarrow e \gamma$ (ICS), $e \gamma_{C M B} \rightarrow e e^{+} e^{-}$(TPP) and $e \gamma_{C M B} \rightarrow e \mu^{+} \mu^{-}$(MPP). The cross section for pion production is small [24], and neglected.

For center of mass energy $s \gg m_{e}^{2}$, the cross sections for PP and ICS are related by $\sigma_{\mathrm{PP}}=2 \sigma_{\mathrm{ICS}}$, with $\sigma_{\mathrm{PP}}$ given by eq. (2). The elasticity of these reactions is

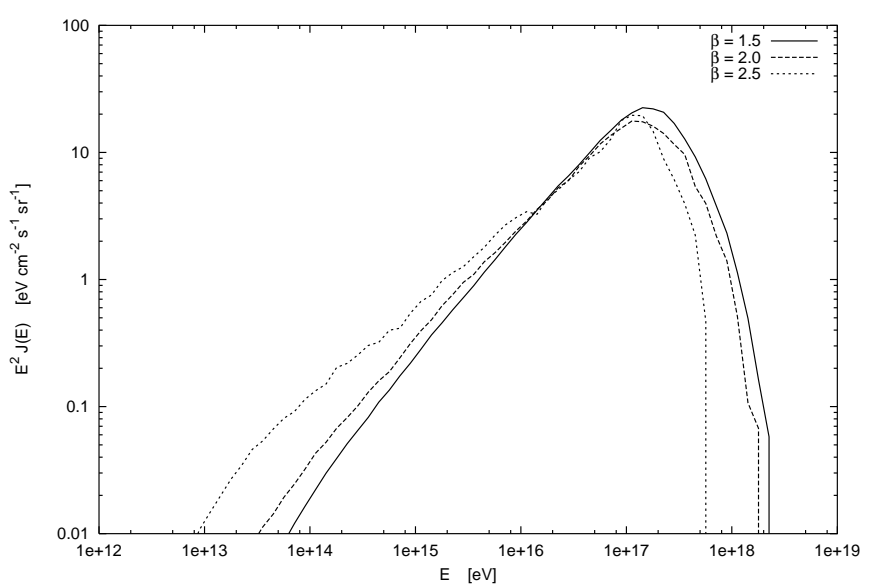

FIG. 2. Differential neutrino flux $E^{2} J(E)$ produced by sources with different photon spectra $n_{\gamma} \propto E_{\gamma}^{-\beta}: \beta=1.5,2$ and 2.5. For all plots $m=3, z_{\max }=10^{3}$, and $z_{\min }=5$.

small: $\eta=1-2\left(\mathrm{~m}^{2} / \mathrm{s}\right)$. For $s \lesssim 100 \mathrm{~m}^{2}$, the high energy approximation is not valid, and one has to resort to the exact (tree level) expressions as can be found in any textbook [29]. The corresponding results for MP can be obtained through the replacement $m_{e} \leftrightarrow m_{\mu}$. The DPP cross section quickly approaches its asymptotic value $\sigma_{\mathrm{DPP}} \simeq 6.45 \mu \mathrm{barn}$. The kinematics of this reaction has not been calculated. We have assumed that the energy of the incoming photon is evenly shared by the produced particles, i.e. four UHE electrons are produced, each with average energy $E_{e}=E_{\gamma} / 4$. This assumption does not affect the outcome much though: using instead that only two of the electrons get all the energy yields a flux that is almost indistinguishable from the flux obtained by the four-electron-assumption.

The most important interactions for neutrino generation are TPP and MPP: more than $95 \%$ of all cascade interactions are TPP, and MPP governs muon production. For $s \gtrsim 100 m_{e}^{2}$ the cross section for TPP is well approximated by the Borsellino formula [26], which in the high-energy limit reduces to eq. (5). The elasticity of the leading electron is very small, eq (4). The cross sections for MPP can be obtained from TPP by replacing $m_{e}$ with $m_{\mu}$, and multiplying by a symmetry factor $S=2$. For lower center of mass energies we used the numerical results obtained in [9]. If energetic enough, the electron produced together with the muon pair in MPP can produce a pair of muons itself; we have incorporated this effect in our computation.

In figures 1 and 2 we have plotted the differential neutrino flux produced by various photon sources. We have assumed that the photon emission rate can be written in the form $\dot{n}_{\gamma}=\dot{n}_{0, \gamma} E_{\gamma}^{-\beta}\left(t / t_{0}\right)^{m}$, and have varied the values of $m$ (figure i1) and $\beta$ (figure 2). For all plots we have taken $z_{\min }=5, z_{\max }=10^{3}$ and photon energies in the range $10^{17} \mathrm{eV}<E_{\gamma}<5 \times 10^{20}$. To normal- 


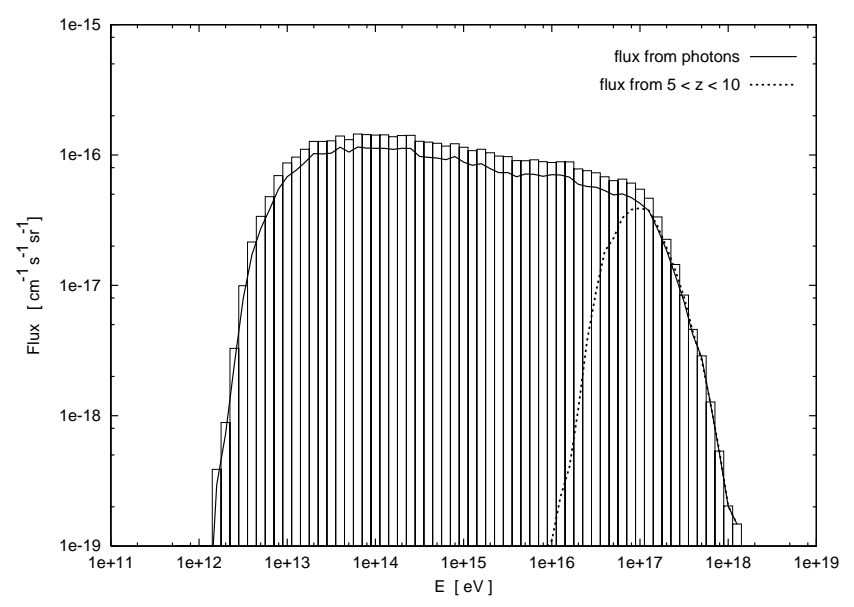

FIG. 3. Neutrino $\phi_{\nu}$ flux produced by necklaces. Area under the solid line is the contribution from primary photons to the flux. Area under the dashed line is the contribution to the flux from red shifts $5<z<10$.

ize the flux, we have assumed that the photon sources are the origin of the UHECR at present, see eq. (10). The results agree well with our estimates. The highest energy neutrinos have energies $E_{\nu} \sim 10^{17} \mathrm{eV}$. At these energies the differential flux is peaked; for $m=3$ sources the peak value is $E_{\nu}^{2} J\left(E_{\nu}\right)=E_{\nu}^{2} \mathrm{~d} \phi_{\nu} / \mathrm{d} E_{\nu} \sim$ $10 \mathrm{eVcm}^{-2} \mathrm{~s}^{-1} \mathrm{sr}^{-1}$. The neutrino flux is smaller for lower values of $m$, and the peak value decreases accordingly: $\left(E^{2} J\right)_{\text {peak }} \sim 1-0.1 \mathrm{eVcm}^{-2} \mathrm{~s}^{-1} \mathrm{sr}^{-1}$ for $m=2$ to $\left(E^{2} J\right)_{\text {peak }} \sim 10^{-3}-10^{-2} \mathrm{eVcm}^{-2} \mathrm{~s}^{-1} \mathrm{sr}^{-1}$ for $m=0$. The flux drops sharply at neutrino energies $E_{\nu} \sim 10^{18} \mathrm{eV}$ just above the peak energies, due to the cutoff at $z=$ $z_{\min }=5$. In reality, the radio background and intergalactic magnetic field are turned on gradually, and the cutoff is somewhat less sharp. The shape of the photon spectrum is not crucial for the results. Values of $\beta=1.5,2,2.5$ all give similar neutrino spectra, with peak values differing by less than a factor $\sim 2$.

We will now discuss in more detail the results for one of the candidate $m=3$ sources, namely necklaces. The UHE photons emitted by necklaces and other topological defects are the result of decaying superheavy "X" particles. For example, X particles may be injected by super conducting strings, emitted from cusps or intersections of ordinary strings, or produced in the annihilation of monopole anti-monopole pair [7] 9]. A general feature of decaying supermassive $\mathrm{X}$ particles is a predominance of pions among the decay products. Charged pions decay through the chain $\pi \rightarrow \mu \bar{\nu}_{\mu} \rightarrow e \bar{\nu}_{e} \nu_{\mu} \bar{\nu}_{\mu}$, producing electrons and neutrinos. This gives a flux of primary neutrinos, peaked at high energies: $E_{\nu} \sim 10^{20} \mathrm{eV}$. The photons emitted by neutral pion decay $\left(\pi^{0} \rightarrow \gamma \gamma\right)$ together with the electrons from charged pion decay also produce a flux of neutrinos, through the mechanism described in section III. In our calculation, we have taken into account both the UHE photons and electrons from

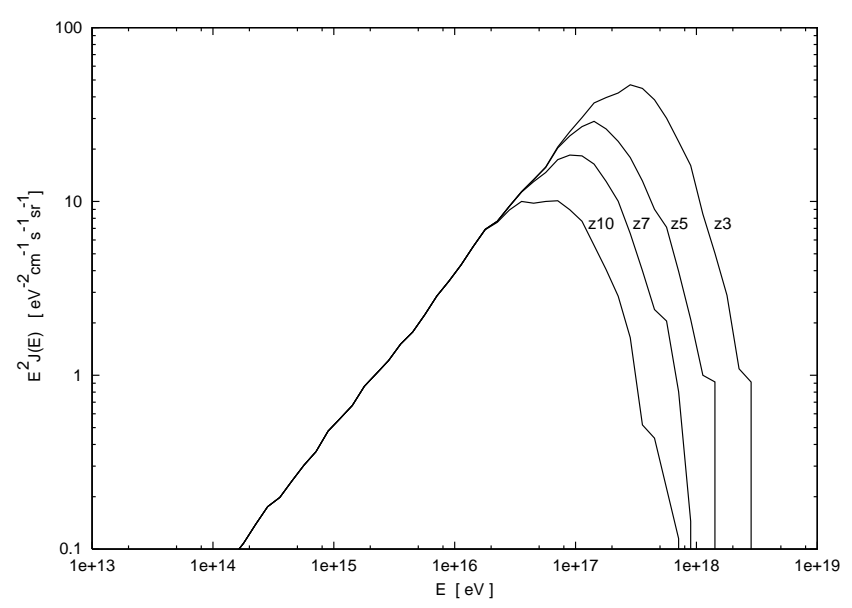

FIG. 4. Differential neutrino flux $E^{2} J(E)$ produced by necklaces for various values of $z_{\min }$. Here $z 3$ corresponds to $z_{\min }=3, z 5$ to $z_{\min }=5, z 7$ to $z_{\min }=7$, and $z 10$ to $z_{\min }=10$.

pion decay. Since the average electron energy is lower than the average photon energy, the UHE photons will give the dominant contribution to the secondary neutrino flux.

The results for necklaces are shown in figures 3 and 4 . In fig. 3 the neutrino flux as function of neutrino energy is plotted, for $z_{\min }=5, z_{\max }=10^{3}$ and photon energies $10^{17} \mathrm{eV}<e_{\gamma}<5 \times 10^{20} \mathrm{eV}$. We used the photons spectrum calculated (and normalized to fit the UHECR data) in [9], extrapolated down to energies as low as $E_{\gamma}=10^{17} \mathrm{eV}$. The contribution from the primary photons to the neutrino flux is indicated by the solid line in the plot. As anticipated, it constitutes the main contribution. The neutrino flux generated at small red shift $5<z<10$ is given by the dashed line. The total flux coming from these red shifts is of the order $\phi_{\nu} \sim 10^{-16} \mathrm{~cm}^{-2} \mathrm{~s}^{-1} \mathrm{sr}^{-1}$, in agreement with our estimate, eq. (12).

The neutrino production mechanism is robust, and the produced flux is not much affected by small changes in cross sections or elasticities. Changes of $10 \%$ in the MPP cross section, the elasticity of the leading particle and of the produced muons do not change the produced flux by more than a factor $\sim 2$. The shape of the photon spectrum gives an uncertainty of the same order or magnitude, as can be seen from fig. 2. Changing the value of $z_{\min }$ makes a more appreciable difference in the produced flux of highest energy neutrinos. Fig. 目shows $E^{2} J(E)$ for values of $z_{\min }=3,5,7$ and 10 . Still, the difference in flux with cutoff $z_{\min }=3$ and cutoff $z_{\min }=10$ is less than a factor 10. For all cutoffs the flux has peak value $E^{2} J(E)=\mathcal{O}(\infty /) \mathrm{eVcm}^{-} \epsilon_{\mathrm{S}^{-\infty}} \mathrm{sr}^{-\infty}$ at the high end of the neutrino spectrum $E_{\nu} \sim 10^{17}-10^{18} \mathrm{eV}$.

The density of $m \geq 4$ sources, such as superconducting strings, is constrained by the EGRET bound. The flux of the cascade photons in the energy range 


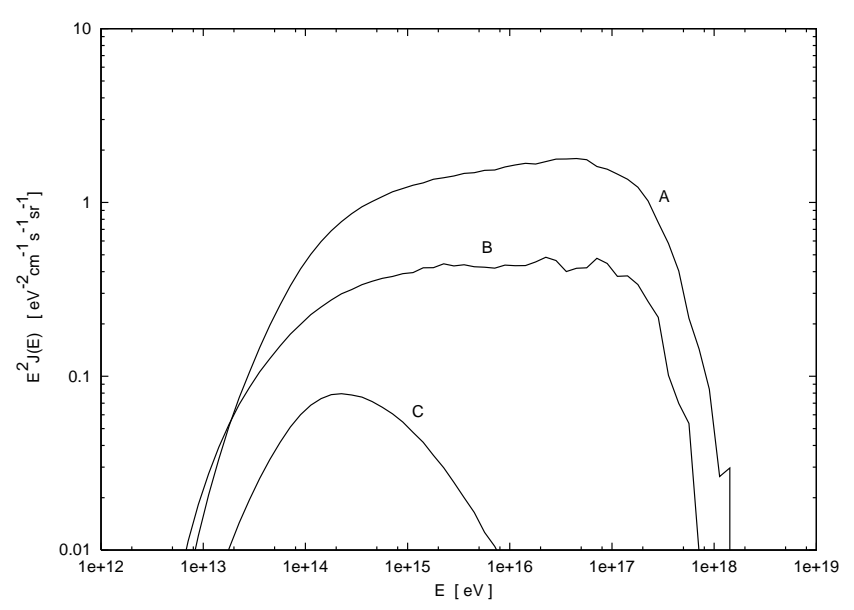

FIG. 5. Maximum differential neutrino flux $E^{2} J(E)$ that can be produced by $m \geq 4$ sources, in accordance with the EGRET bound. Line A corresponds to $m=4$ and $\beta=1.5$ photon spectrum, line $\mathrm{B}$ to $m=4$ and $\beta=2$, and line $\mathrm{C}$ to $m=5$ and $\beta=2.5$.

$10 \mathrm{MeV}<E<100 \mathrm{GeV}$ must be lower than the flux measured: $\omega_{\text {cas }} \leq 2.6 \times 10^{-6} \mathrm{eVcm}^{3}$. One can estimate the flux of cascade photons produced by TD's by assuming that all the energy density emitted through UHE photons is transferred to the low energy photons. The universe becomes transparant for low energy photons at red shift $z \sim 10^{3}$. The EGRET bound then constrains the photon flux to be

$$
\int_{z=0}^{z_{\mathrm{GZK}, \gamma}} \mathrm{d} z \partial J\left(E_{\gamma}, z\right) / \partial E_{\gamma} \lesssim\left(10^{-2}-10^{-3}\right) \phi_{\mathrm{CR}} .
$$

The maximum flux of neutrinos that can be produced by $m \geq 4$ sources when this bound is taken into account is $E^{2} J(E) \sim \mathcal{O}(\infty)$, as shown in figure 3. Here we used $z_{\min }=5, z_{\max }=10^{3}$ and photon energies in the range $10^{17} \mathrm{eV}<e_{\gamma}<5 \times 10^{20} \mathrm{eV}$. The photon spectrum is taken to be of the form $\dot{n}_{\gamma, 0} \propto E_{\gamma}^{-\beta}$. Flux A corresponds to $m=4$ and $\beta=1.5$, flux B to $m=4$ and $\beta=2$. For $m \geq 4$ sources the EGRET constraint is more severe. Flux $\mathrm{C}$ shows the result for $m=5$ and $\beta=1.5$.

\section{DISCUSSION}

Neutrinos from slowly decaying relic particles or some other $m \leq 2$ source are too sparse to be detectable in the foreseeable future. However, the flux from $m=3$ sources, such as necklaces, may be detected soon. The neutrino spectrum generated by $m=3$ sources has a peak value $\left(E^{2} J\right)_{\text {peak }}=\mathcal{O}(\infty \prime) \mathrm{eVcm}^{-} \epsilon_{\mathrm{s}}{ }^{-\infty} \mathrm{sr}^{-\infty}$ for neutrino energies $E_{\nu} \sim 10^{17}-10^{18} \mathrm{eV}$. In all our calculations we assumed that the photon sources in question are resposible for the UHECR today. Certainly, the flux of photons emitted cannot exceed the observed UHECR flux, and thus our calculations can be interpreted as an upper bound on the flux of secondary neutrinos produced.

Can other sources produce a comparable flux of neutrinos at $10^{17}-10^{18} \mathrm{eV}$ ? The neutrino flux $\phi_{\nu} \sim$ $10^{-16} \mathrm{~cm}^{-2} \mathrm{~s}^{-1} \mathrm{sr}^{-1}$ at $E_{\nu} \sim 10^{17} \mathrm{eV}$ exceeds the background flux from the atmosphere and from pion photoproduction on CMBR at this energy 11, 15, 31, as well as the fluxes predicted by a number of models [19]. Models of active galactic nuclei (AGN) have predicted a similar flux of neutrinos at these energies [6]. The predictions of these models have been a subject of debate [32]. However, the neutrinos produced by photon sources at high red shift have a distinctive feature: they create a sharp "bump" in the spectrum. Moreover, if the photon sources are TD's the secondary flux is accompanied by a primary neutrino flux peaked at $E_{\nu} \sim 10^{20} \mathrm{eV}$. And everyone agrees that AGN cannot produce neutrinos with energies of $10^{20} \mathrm{eV}$ 33]. So, an observation of $10^{17} \mathrm{eV}$ neutrinos accompanied by a comparable flux of $10^{20} \mathrm{eV}$ neutrinos would be a signature of a TD rather than an AGN.

The density of TD's with $m \geq 4$, e.g. superconducting strings, is constrained by the EGRET bound on the flux of low energy $\gamma$-photons. This also constrains the neutrino flux these sources can produce to be less than $E^{2} J(E) \leq \mathcal{O}(\infty) \mathrm{eVcm}^{-} \mathrm{s}^{-\infty} \mathrm{sr}^{-\infty}$. This is probably too low to be detected.

To summarize, we have shown that sources of ultrahigh energy photons that operate at red shift $z \gtrsim 5$ produce neutrinos with energy $E_{\nu} \sim 10^{17} \mathrm{eV}$. The flux depends on the evolution index $m$ of the source. A distinctive characteristic of this type of neutrino background is a "bump" in the spectrum at neutrino energies $E_{\nu}=10^{17}-10^{18} \mathrm{eV}$, and a sharp cutoff at $E_{\nu} \sim 10^{18} \mathrm{eV}$ due to the universal radio background at $z<z_{\min }$. We have calculated numerically the neutrino spectrum produced by various sources. The produced flux is largest for $m=3$ sources. It has a peak value $E^{2} J(E)=\mathcal{O}(\infty /) \mathrm{eVcm}^{-} \epsilon_{\mathrm{s}}{ }^{-\infty} \mathrm{sr}^{-\infty}$ at neutrino energies $E_{\nu} \sim 10^{17}-10^{18} \mathrm{eV}$. Detection of these neutrinos can help understand the origin of ultrahigh energy cosmic rays. Sources with larger values of the evolution index $m \geq 4$ are ruled out as the origin of observed UHECR by the EGRET bound on the flux of $\gamma$-photons. This bound also constrains the flux from these sources to be less than $\left(E^{2} J\right)_{\text {peak }} \leq$ $\mathcal{O}(\infty) \mathrm{eVcm}^{-\epsilon} \mathrm{s}^{-\infty} \mathrm{sr}^{-\infty}$.

The author would like to thank A. Kusenko and C. Postma for useful discussions. This work was supported in part by the US Department of Energy grant DE-FG03-91ER40662, Task C, and by a grant from UCLA Council on Research. 
[1] A. Kusenko and M. Postma, Phys. Rev. Lett. 86, 1430 (2001).

[2] M. Takeda et al., Phys. Rev. Lett. 81, 1163 (1998); M.A. Lawrence, R.J. Reid and A.A. Watson, J. Phys. G G17, 733 (1991); D. J. Bird et al., Phys. Rev. Lett. 71, 3401 (1993); Astrophys. J. 424, 491 (1994).

[3] K. Greisen, Phys. Rev. Lett. 16, 748 (1966); G. T. Zatsepin and V. A. Kuzmin, Pisma Zh. Eksp. Teor. Fiz. 4, 114 (1966).

[4] For review, see, e.g., P. L. Biermann, J. Phys. G G23, 1 (1997); P. Bhattacharjee and G. Sigl, Phys. Rept. 327, 109 (2000).

[5] E. Waxman, Phys. Rev. Lett. 75, 386 (1995); G. R. Farrar and P. L. Biermann, Phys. Rev. Lett. 81, 3579 (1998); A. Dar, A. De Rujula and N. Antoniou, astroph/9901004; G. R. Farrar and T. Piran, Phys. Rev. Lett. 84, 3527 (2000); E. Ahn, G. Medina-Tanco, P. L. Biermann, and T. Stanev, astro-ph/9911123.

[6] K. Mannheim, R. J. Protheroe and J. P. Rachen, Phys. Rev. D, to appear astro-ph/9812398; astro-ph/9908031.

[7] A. Vilenkin, Phys. Rept. 121, 263 (1985); A. Vilenkin and E. P. S. Shellard, Cosmic strings and other topological defects, Cambridge University Press, Cambridge, England, 1994; M. B. Hindmarsh and T. W. Kibble, Rept. Prog. Phys. 58, 477 (1995).

[8] C. T. Hill and D. N. Schramm, Phys. Rev. D31, 564 (1985); C. T. Hill, D. N. Schramm, and T. P. Walker, Phys. Rev. D36, 1007 (1987); P. Bhattacharjee, C. T. Hill, and D. N. Schramm, Phys. Rev. Lett. 69, 567 (1992).

[9] V. Berezinsky, P. Blasi and A. Vilenkin, Phys. Rev. D58, 103515 (1998).

[10] V. S. Berezinsky and A. Vilenkin, hep-ph/9908257.

[11] V. Berezinsky, M. Kachelriess, and A. Vilenkin, Phys. Rev. Lett. 79, 4302 (1997); V. A. Kuzmin and V. A. Rubakov, Phys. Atom. Nucl. 61, 1028 (1998) [Yad. Fiz. 61, 1122 (1998)]; V. Kuzmin and I. Tkachev, JETP Lett. 68, 271 (1998); M. Birkel and S. Sarkar, Astropart. Phys. 9, 297 (1998); D.J. Chung, E.W. Kolb, and A. Riotto, Phys. Rev. Lett. 81, 4048 (1998); Phys. Rev. D59, 023501 (1999); K. Benakli, J. Ellis, and D. V. Nanopoulos, Phys. Rev. D59, 047301 (1999); Phys. Rev. D59, 123006 (1999).

[12] G. Gelmini and A. Kusenko, Phys. Rev. Lett. 84, 1378 (2000). J. L. Crooks, J. O. Dunn, and P. H. Frampton, astro-ph/0002089.

[13] For review, see, e.g., V. A. Kuzmin and I. I. Tkachev, Phys. Rept. 320, 199 (1999).

[14] C. T. Hill and D. N. Schramm, Phys. Lett. B131, 247 (1983).

[15] F. W. Stecker, C. Done, M. H. Salamon, and P. Sommers, Phys. Rev. Lett. 66, 2697 (1991); erratum: ibid., 69, 2738 (1992).

[16] S. Yoshida, G. Sigl, and S. Lee, Phys. Rev. Lett. 81, 5505 (1998). G. Sigl, S. Lee, P. Bhattacharjee, and S. Yoshida, Phys. Rev. D59, 043504 (1999)

[17] R. J. Protheroe and P. A. Johnson, Astropart. Phys. 4, 253 (1996) astro-ph/9506119.

[18] R. J. Protheroe and T. Stanev, Phys. Rev. Lett. 77, 3708 (1996) astro-ph/9605036].

[19] D. B. Cline and F. W. Stecker, astro-ph/0003459.
[20] V. Berezinsky, Sov. J. of Nucl. Phys. 11, 222 (1970).

[21] R. J. Protheroe and P. L. Biermann, Astropart. Phys. 6, 45 (1996).

[22] J. J. Condon, Astrophys. J. 284, 44 (1984).

[23] P. Madau et al., Mon. Not. R. Astron. Soc. 283, 35 (1996); C.C. Steidel, Proc. Nat. Acad. Sci. 96, 4232, 1999; T. Miyaji, G. Hasinger, and M. Schmidt, astroph/9809398; G. Pugliese, H. Falcke, Y. Wang, and P.L. Biermann, Astron. and Astrophys., 358, 409 (2000).

[24] J. Boyer et al., Phys. Rev. D42, 1350 (1990).

[25] R. W. Brown, W. F. Hunt, K. O. Mikaelian and I. J. Muzinich, Phys. Rev. D8, 3083 (1973).

[26] A. Borsellino, Nuovo Cimento 4, 112 (1947); K. J. Mork Phys. Rev. 160, 1065 (1967); A. Mastichiadis, A. P. Marscher and K. Brecher.

[27] A. Mastichiadis, A. P. Marscher, and K. Brecher, Astrophys. Journal 300, 178 (1986); V. Anguelov, S. Petrov, L. Gurdev and J. Kourtev, J. Phys. G25, 1733 (1999).

[28] P. Gondolo, G. Gelmini and S. Sarkar, Nucl. Phys. B392, 111 (1993).

[29] M. E. Peskin and D. V. Schroeder, An introduction to quantum field theory, Addison-Wesley Publishing Company, 1995.

[30] EGRET collaboration, P. Streekumar et al., Astrophys. J. 494, 523 (1998).

[31] T. Stanev, R. Engel, A. Muecke, R. J. Protheroe and J. P. Rachen, Phys. Rev. D62, 093005 (2000) astro$\mathrm{ph} / 0003484$.

[32] E. Waxman and J. Bahcall, Phys. Rev. D59, 023002 (1999).

[33] J. P. Rachen and P. Mészáros, Phys. Rev. D58, 123005 (1998) astro-ph/9802280. 